\title{
Lung ultrasound in cardiology: Realities and promises
}

\section{El ultrasonido pulmonar en cardiología: realidades y promesas}

\author{
Rodrigo Gopar-Nieto', Gabriela P. Alanís-Estrada², Diestefano E. Ronquillo-Ramírez², \\ Jorge L. Vargas-Estrada², Alexandra Arias-Mendoza², Gustavo Rojas-Velasco ${ }^{3}$, and \\ Diego Araiza-Garaygordobil**
}

${ }^{1}$ Department of Cardiology; ${ }^{2}$ Coronary Care Unit; ${ }^{3}$ Post-surgical Therapy. Instituto Nacional de Cardiología Ignacio Chávez, Secretaría de Salubridad, Mexico City, Mexico

\begin{abstract}
Lung ultrasound (LUS) is an easily available, reproducible examination tool, both in ambulatory and critically ill patients, which is used to evaluate congestion status and to differentiate the etiology of dyspnea. In this review, we explain LUS technique, acquisition protocols and their interpretation, as well as the evidence that show its effectiveness among stable and critically ill patients. LUS should be used as an add-on to traditional physical examination to give an accurate diagnosis and a rapid treatment to patients with pulmonary congestion.
\end{abstract}

Key words: Lung ultrasound. Heart failure. Dyspnea.

\section{Resumen}

El ultrasonido pulmonar es una herramienta de fácil reproducibilidad en pacientes estables y críticos que se utiliza para valorar el estado de congestión y comprobar la causa de la disnea. En este texto se revisan los fundamentos de la ecografía pulmonar, los protocolos de adquisición y su interpretación, así como la evidencia que sustenta su uso en el paciente cardiovascular críticamente enfermo y en el paciente estable. Esta herramienta debe emplearse como complemento de la exploración física regular para poder instituir un tratamiento oportuno en los pacientes con congestión pulmonar.

Palabras clave: Ultrasonido pulmonar. Insuficiencia cardíaca. Disnea.

\section{Correspondence:}

*Diego Araiza-Garaygordobil

Juan Badiano, 1

Col. Belisario Domínguez, Sección XVI, Del. Tlalpan

C.P. 14080 , Ciudad de México, México

Date of reception: 04-04-2019

Date of acceptance: 04-06-2019

DOI: $10.24875 /$ ACME.M20000070
Available online: $05-08-2019$ Arch Cardiol Mex (Eng). 2019;89(4):338-343 www.archivoscardiologia.com 2604-7063/@ 2019 Instituto Nacional de Cardiología Ignacio Chávez. Published by Permanyer. This is an open access article under the CC BY-NC-ND license (http://creativecommons.org/licenses/by-nc-nd/4.0/). 


\section{Introduction}

Lung ultrasound (LUS) is a focused, quick and widely available technique that is harmless to the patient and easy to reproduce for the operator and has started to form part of cardiologists' tools since one decade ago. Nevertheless, it is still far from being systematically applied in all cardiovascular medicine scenarios, particularly due to ideological barriers, such as those established in internal medicine classical texts of the end of the $20^{\text {th }}$ century ${ }^{1}$, which claimed that the lung is an obstacle to thoracic imaging. However, works from several authors, especially Lichtenstein, showed the usefulness of lung echography as a tool in the treatment of the patient with critical conditions and, subsequently, the bases for its use in the stable and decompensated cardiovascular patient were established ${ }^{2}$, to such an extent that guidelines such as those of the European Society of Cardiology recommend the use of LUS in acute heart failure with a grade of evidence $\mathrm{IIB}^{3}$.

With the increasing availability of pocket-sized ultrasound scanners, lung and other organs echography should be increasingly applied in daily clinical practice. The purpose of this review is to describe the fundamentals of LUS, the acquisition protocols and their interpretation, as well as the evidence that support its use in critically ill cardiovascular patients and in stable subjects. Finally, the knowledge gaps that need to be solved by research studies to more efficiently implement this technique in cardiologists' daily practice are shown.

\section{LUS fundamentals}

LUS is based on the observation of a series of artifacts produced by the interaction between ultrasound and pleural and alveolar interfaces and alveolar air. There are several conditions that can alter this interaction and cause pathological effects, including the presence of fluid, air, alveolar edema, or pulmonary consolidation, which can be identified as a series of ultrasound findings that project at least 10 signs that enable ultrasonographic examination of the lung. On average, an LUS examination can be carried out in 1 $m$ or less, and the information obtained can help to rule out the presence of pleural effusion or pulmonary edema or suggest other conditions such as pneumothorax, pneumonia or consolidation, or COPD, among others. The acquisition technique and interpretation can be standardized in 1-day sessions or with teaching through digital modules available online ${ }^{4}$.

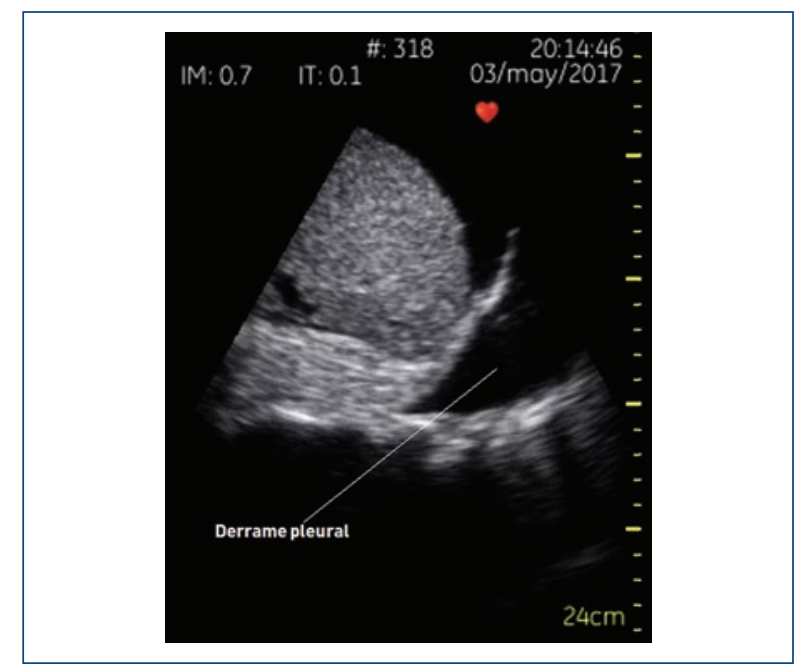

Figure 1. Lung ultrasound with pleural effusion image. Derrame pleural $=$ pleural effusion .

\section{Acquisition technique}

As for the acquisition technique, it should be emphasized that the transducer must be placed perpendicularly to the chest wall obliquely following the direction of the ribs. A $3.5-5 \mathrm{MHz}$ transducers allow adequate visualization of subpleural structures, and it should be noted that linear transducers detail pleura better, while cardiac transducers enable better visualization in terms of depth and pulmonary alterations ${ }^{4,5}$.

One of the great advantages is that an acoustic window is almost always available, even in cases where performing a transthoracic echocardiogram is not possible. In other words, LUS is feasible in obese patients, in critically ill individuals in the supine position, with invasive mechanical ventilation and hemodynamically unstable. For uniform and reproducible easy views, having three symmetric regions per pulmonary field have been proposed, two in the anterior part and one posterolateral. Finally, it is very important mentioning that the posterolateral point serves to corroborate the presence of pleural effusion with a sensitivity of $>90 \%$ (Fig. 1) 6 .

It is of particular interest for the cardiologist to detect the changes caused by fluid accumulation in the pleural space or pulmonary parenchyma. In both cases, LUS has better diagnostic performance that chest X-ray and serves to start the approach and differentiate the causes of dyspnea, such as pneumonia, with a sensitivity and specificity of 89 and $94 \%$, respectively, in the presence of alveolar consolidation and a diffuse pattern of anterior B-lines without pleural sliding, pleural effusion 


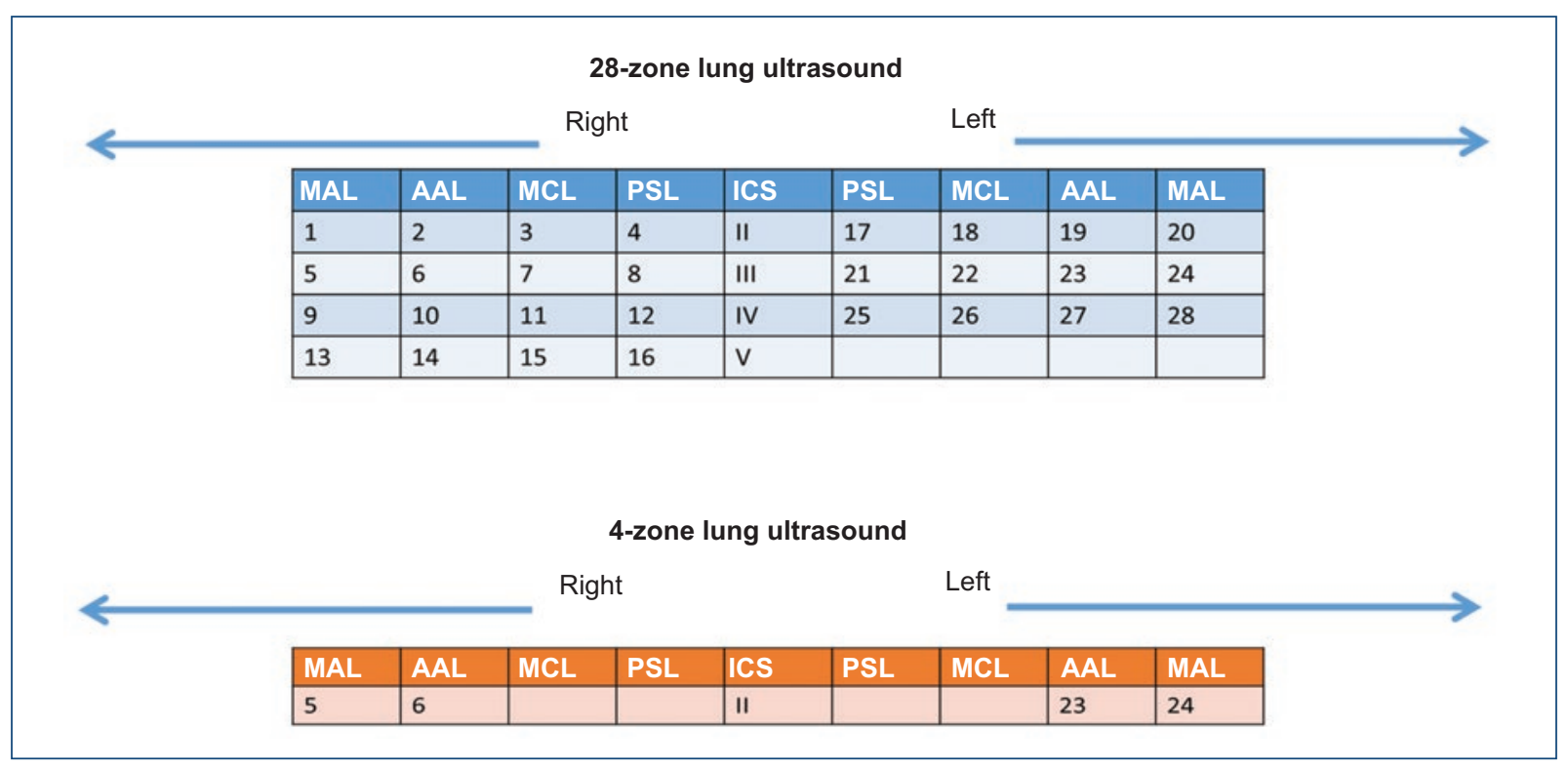

Figure 2. Comparison between 28- and 4-zone lung ultrasound acquisition protocols.

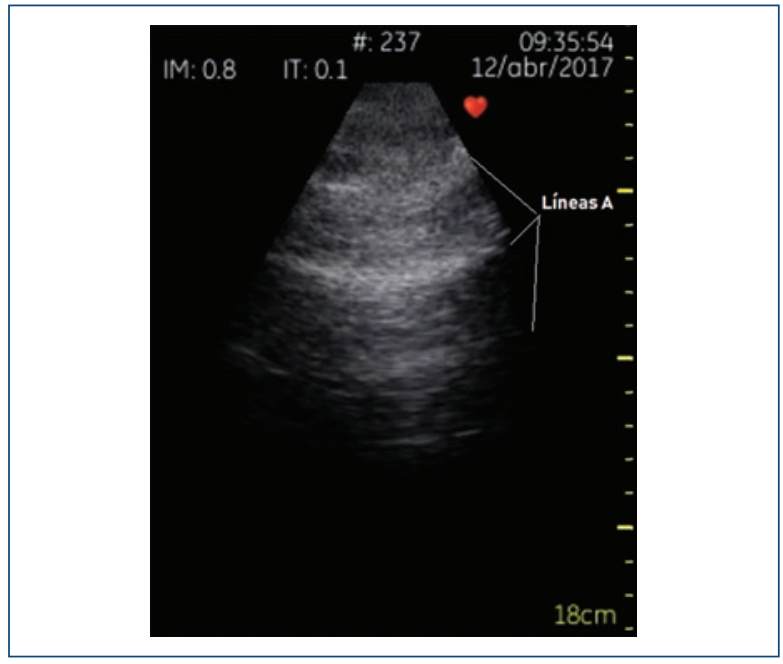

Figure 3. A-lines visualized with lung ultrasound. Líneas $A=$ A-lines.

(shows higher accuracy in comparison with portable chest X-ray, $93 \%$ vs. $47 \%$ ), acute pulmonary edema in the presence of diffuse anterior B-lines with lung sliding (97\% sensitivity and $94 \%$ specificity), as well as in chronic obstructive pulmonary disease, asthma, pulmonary thromboembolism, and pneumothorax ${ }^{7}$.

For LUS acquisition, the depth of the device must be adjusted according to patient complexion, with less depth being required in thin patients and higher in obese subjects, to enable properly visualizing the

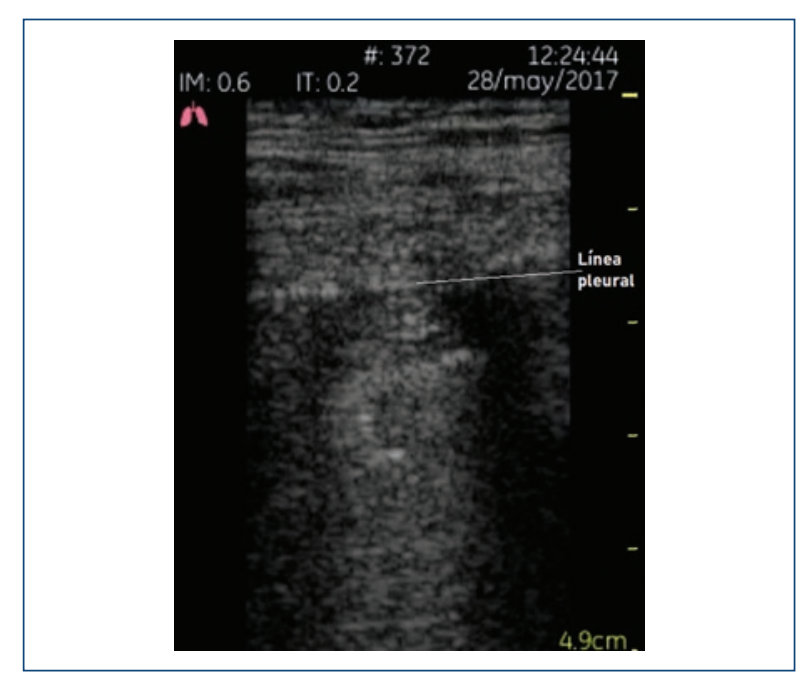

Figure 4. Pleural line in lung ultrasound with linear transducer.

Línea pleural = pleural line.

pleural line. In the world literature, at least five types of protocols for LUS image acquisition have been described, which include 28-, 16-, 8-, 4-, and 1-zone images $^{8}$. More extensive protocols (28 zones) have been compared with abbreviated protocols $(3,4$, and 8 zones) for diagnostic capacity, with abbreviated protocols area under the curve being larger than 0.9 to detect common pathological phenomena such as congestion, considering that the presence of 3 or more B-lines per field, in two or more fields per hemithorax, 


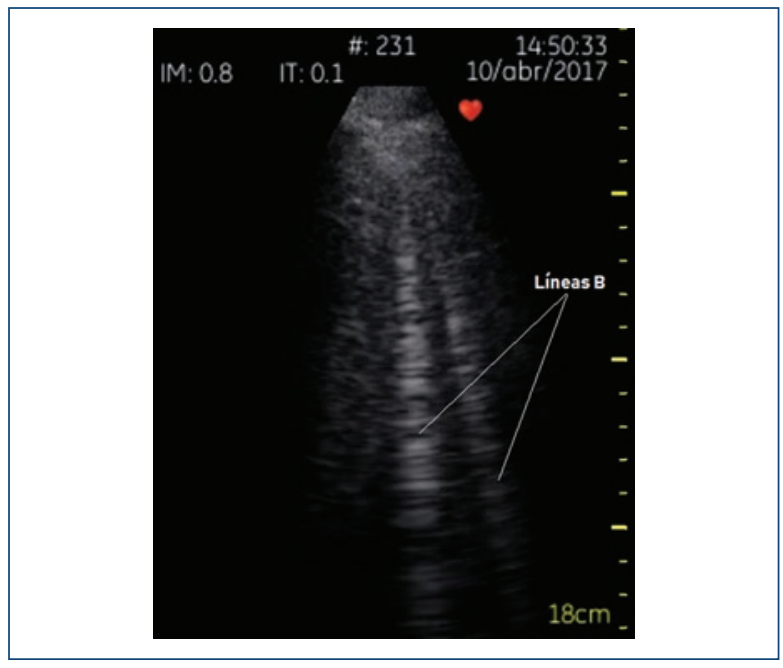

Figure 5. Multiple B-lines with lung ultrasound. Líneas $B=\mathrm{B}$-lines.

in both hemithoraces, defines the existence of acute pulmonary edema ${ }^{7}$, pneumothorax, or effusion, and the use of the latter has, therefore, been proposed for faster visualization, without the loss of relevant data (Fig. 2).

\section{Main signs in LUS}

Ultrasound normal interaction with the lung causes an artifact known as A-lines (Fig. 3). These are part of a macroscopically normal lung and are produced by the echo of both pleurae (parietal and visceral pleura), which form a "pleural line." This is visible just a few centimeters below the ultrasound transducer and, in turn, shows the normal "sliding" movement produced by breathing (Fig. 4), which has an amplitude of 10-15 $\mathrm{mm}^{9}$. The echo produced by the pleural line is, in turn, visible and forms A-lines, which constitute another normal lung finding and is found at equidistant distances between the transducer and the pleural line, and can be found at a rate of one, two, three, or more in a healthy lung. The presence of pleural line with pleural sliding and A-lines indicates a healthy lung. Abnormal patterns that can be found occur in two situations: (a) when there is air between the pleural layers, i.e., in case of pneumothorax, which results in the movement disappearing and (b) when there is exudate between both layers, as in infectious processes, with the movement decreasing or not existing.

The fundamental event that causes cardiac failure is filling pressure increase and cardiogenic pulmonary edema. Cardiogenic alveolar edema which is the consequence of the rise in diastolic left atrium pressures for different reasons (left ventricular systolic dysfunction, diastolic, or both, mitral valve stenosis or regurgitation, left ventricular outflow tract obstruction for different reasons, left ventricular acute ischemia, etc.) is related to alveolar-capillary interface increased thickness. This process, in addition to causing dyspnea, decreased oxygenation, cough, and other symptoms, produces characteristic acoustic artifacts, known as B-lines or comet-tail artifacts due to their resemblance with that astronomical object.

B-lines are shaped as a vertically-oriented comet, they originate in the pleural line and move synchronically with pulmonary sliding. Quite often they are defined as laser shaped, they erase the A-lines and tend to be hyperechoic (Fig. 5). One, two, or more B-lines can be identified within the same ultrasonographic field; sometimes, they are recognized as individual and differentiable and they fuse to form something that is known as a "B" pattern, which is characterized for occupying a percentage of the scan area and for being constituted of multiple merged $\mathrm{B}$-lines ${ }^{4-10}$. The number of $\mathrm{B}$-lines is a parameter of severity and has been accompanied by a poorer prognosis, as described below.

\section{LUS and heart failure and dyspnea}

Heart failure, either with diminished or preserved ejection fraction, is a fundamental entity in cardiovascular pathology and in cardiologists' daily practice. Usually, heart failure diagnosis has been based on findings on physical examination and patient history. However, as the prevalence of heart failure increases, the need to have clinical tools with a superior diagnostic and differentiating power to that of clinical examination (such as echocardiogram and brain and atrial natriuretic peptides) has become evident.

LUS can be considered a reliable tool for the assessment of pulmonary congestion in patients with heart failure because it constitutes an extension of physical examination and helps to differentiate pulmonary congestion hemodynamics.

Within the most recent advances, attempts have been made to optimize LUS-guided medical treatment and showing that a significant improvement was possible regarding the sensation of dyspnea (7.68 vs. 3.57 , $p=0.007$, as measured with the dyspnea visual analog scale) within the first $12 \mathrm{~h}$ of treatment; it should be further mentioned that treatment response is related to long-term survival, which shows a risk decrease (hazard ratio [HR]) of 0.19 (95\% confidence interval [Cl], 
0.06-0.67, $p=0.010$ ) in comparison with all-cause mortality ${ }^{11,12}$. This technique is attractive for outpatient clinical assessment because it uses simple technology, where pocket devices stand out, and it is quick to be performed and interpreted. As a possible clinical implication, pharmacotherapy could be started as soon as the patient shows a significant increase in the number of B-lines, even if asymptomatic ${ }^{13}$. Finally, it should be mentioned that pulmonary congestion has also been studied in special populations, such as people on hemodialysis, where it has been used to detect asymptomatic congestion, with its presence being found to be a predictor of death and cardiac episodes; this highlights the importance of using this tool in an early manner ${ }^{14}$.

On the other hand, in the context of compensated chronic heart failure, pulmonary congestion evaluation has usually been performed with physical examination and chest X-ray but, more recently, LUS has been shown a sensitive and semi-quantitative tool. It is in this aspect where it has shown that the presence of three or more B-lines is correlated with adverse cardiovascular outcomes, in particular with a higher risk of rehospitalization and mortality ${ }^{15-17}$. Meanwhile, in patients with decompensated heart failure, LUS has been used as a predictor of new hospital admissions after the implementation of medical treatment and before discharge, as demonstrated by Gargani et al. In their study, they found that the presence of $>50$ B-lines at admission was the most important predictor of hospital readmission for heart failure during follow-up, with a sensitivity of $71.4 \%$, specificity of $69.8 \%$, negative predictive value of $93.7 \%$, and positive predictive value of $27.8 \%$ (area under the curve of $0.71,95 \% \mathrm{Cl}, 0.58-0.85$, $\mathrm{p}=0.011)^{18}$.

Acute dyspnea is a common cause of hospitalization, and it can occur in multiple situations, including decompensated acute heart failure, chronic obstructive pulmonary disease exacerbation, states of anxiety, and pulmonary embolism. Despite the existence of paraclinical modern methods, the differentiation between dyspnea cardiac and non-cardiac causes is still a clinical problem. LUS has acquired relevance, as the amount of pulmonary ultrasound comets has been shown to increase with the worsening of the New York Heart Association functional class. In addition, correlation has been found with Kerley's B-lines and chest $\mathrm{X}$-ray lung water score, pulmonary extravascular water invasively measured with the thermodilution method and severity of the diastolic dysfunction. A recent meta-analysis compared the sensitivity and accuracy of LUS in comparison with chest X-ray in patients with acute heart failure, including six studies and a total of 1827 patients. The study reported a sensitivity for LUS of $0.88(95 \% \mathrm{Cl}, 0.75-0.95)$ and specificity of $0.90(95 \% \mathrm{Cl}, 0.88-0.92)$, in comparison with a sensitivity of $0.73(95 \% \mathrm{Cl}, 0.70-0.76)$ for chest $X$-ray, with no differences in terms of specificity ${ }^{19}$. In another study, LUS diagnostic performance was compared against natriuretic peptides, with the main causes of cardiogenic dyspnea being observed to be systolic dysfunction, isolated diastolic dysfunction, severe heart valve disease, and arrhythmias. NT-proBNP values were positively correlated with the number of B-lines ( $r=0.69, p<0001)$. The ROC analysis showed that an NT-proBNP plasma concentration of $298 \mathrm{ng} / \mathrm{L}$ had a sensitivity of $97 \%$ and specificity of $92.6 \%$, while the presence of nine B-lines had a sensitivity of $73 \%$ and specificity of $100 \%$. This study demonstrated that B-lines are a simple and useful method for acute cardiogenic versus non-cardiogenic dyspnea differential diagnosis, given that they provide a direct and morphological image that is easily distinguishable from abnormal increases of water in the lungs $^{20}$.

\section{Perspectives and limitations}

Contemporary clinical approach needs to be grounded in the experience collected over the centuries and be combined with new elements that are generated thanks to technological advances. Proof of this is the current availability of pocket-sized ultrasound devices, which allows having a better patient initial assessment. In spite of this, it should not be assumed that new technologies will completely replace usual methods since physical examination will always have an essential place in the doctor-patient relationship and will be a point of reference for medical actions.

Among the limitations of LUS, it should be noted that the only alteration that can cause difficulties to perform a good examination is serious subcutaneous emphysema. On the other hand, since it is a relatively novel assessment tool with applications in very different branches of medicine, its application can cause confusion because it is necessary to define cutoff values and strict methodologies to spread its use.

As for new trends, it can be claimed that today, there are several studies trying to answer the questions about B-lines cutoff point under stress and at rest in patients with heart failure or coronary heart disease, in addition to assess whether to guide therapeutics with 
LUS in patients with acute ${ }^{21}$ and chronic ${ }^{22}$ heart failure is equal to or better than the treatment guided by findings on physical examination. In the population of this country, the CLUSTER-HF trial stands out, which is the first Latin American study on registration phase, and is seeking to establish if LUS will be useful to guide medical treatment in Mexican patients with heart failure (NCT03613779) ${ }^{22}$.

\section{Conclusions}

LUS is a tool that complements the cardiologist's diagnostic imaging armamentarium and helps to quickly and efficaciously differentiate pulmonary overload and the cause of dyspnea in critical patients and outpatients. One of the strengths of this test is its great reproducibility and short acquisition time, which can contribute to continue with its implementation as a tool for diagnostic approach in all cardiologists.

\section{Conflicts of interest}

There are no conflicts of interest.

\section{Funding}

The authors received no funding for the preparation of this document.

\section{Ethical disclosure}

Protection of people and animals. The authors declare that no experiments were performed on humans or animals for this study.

Confidentiality of data. Los autores declaran que han seguido los protocolos de su centro de trabajo sobre la publicación de datos de pacientes.

Right to privacy and informed consent. The authors have obtained the written informed consent of the patients or subjects mentioned in the article. The corresponding author is in possession of this document.

\section{References}

1. Longo D, Fauci AS, Kasper DL, Hauser SK, Jameson JL, Loscalzo J, editors. Harrison's principles of internal medicine. $18^{\text {th }}$ ed. New York: McGraw-Hill, 2011. Part 11, section 1, chapter 253.
2. Lichtenstein D, Mézière G, Biderman $P$, Gepner A, Barré O. The comet-tail artifact. An ultrasound sign of alveolar-interstitial syndrome. Am J Respir Crit Care Med 1997;156:1640-6.

3. Ponikowski $P$, Voors AA, Anker SD, Bueno H, Cleland JGF, Coats AJS et al, ESC Scientific Document Group, 2016 ESC Guidelines for the diagnosis and treatment of acute and chronic heart failure: the task force for the diagnosis and treatment of acute and chronic heart failure of the European Society of Cardiology (ESC) Developed with the special contribution of the Heart Failure Association (HFA) of the ESC, European Heart Journal 2016;372129-2200, https://doi.org/10.1093/eurheartj/ ehw128

4. Picano E, Scali MC, Ciampi Q, Lichtenstein D. Lung ultrasound for the cardiologist. J Am Coll Cardio Ilmg. 2018;11:1692-705.

5. Lichtenstein D. The probe. In: Lung Ultrasound in the Critically III - The BLUE-Protocol. Heidelberg, Germany: Springer, 2016; p 23-35.

6. Lichtenstein D, Lascols N, Méziére G, Gepner A. Ultrasound diagnosis of alveolar consolidation in the critically ill. Intensive Care Med. 2004; 30:276-81.

7. Lichtenstein $D$, Méziére $G$. Relevance of lung ultrasound in the diagnosis of acute respiratory failure: the BLUE protocol. Chest. 2008;134:117-25.

8. Scali MC, Zagatina A, Simova I, Zhuravskaya N, Ciampi Q, Paterni M, et al. B-lines with lung ultrasound: the optimal scan technique at rest and during stress. Ultrasound Med Biol. 2017;43:2558-2566.

9. Lichtenstein D. Lung ultrasound in the critically ill. Ann Intensive Care. 2014;4:1.

10. Xirouchaki N, Kondili E, Prinianakis G, Malliotakis P, Georgopoulos D. Impact of lung ultrasound on clinical decision making in critically ill patients. Intensive Care Med. 2014 Jan;40(1):57-65.

11. Öhman J, Harjola VP, Karjalainen P, Lassus J. Assessment of early treatment response by rapid cardiothoracic ultrasound in acute heart failure: Cardiac filling pressures, pulmonary congestion and mortality. Eur Heart J Acute Cardiovasc Care. 2018;7(4):311-320.

12. Öhman J, Harjola VP, Karjalainen P, Lassus J. Focused echocardiography and lung ultrasound protocol for guiding treatment in acute heart failure. ESC HeartFail. 2018;5(1):120-128.

13. Miglioranza MH, Gargani L, Sant'Anna RT, Rover MM, Martins VM, Mantovani A, et al. Lung ultrasound for the evaluation of pulmonary congestion in outpatients: a comparison with clinical assessment, natriuretic peptides, and echocardiography. JACC Cardiovasc Imaging. 2013; 6(11):1141-51.

14. Zoccali C, Torino C, Tripepi R, Tripepi G, D'Arrigo G, Postorino M, et al. Pulmonary congestion predicts cardiac events and mortality in ESRD. J Am Soc Nephrol. 2013 Mar;24(4):639-46.

15. Platz E, Lewis EF, Uno H, Peck J, Pivetta E, Merz AA, et al. Detection and prognostic value of pulmonary congestion by lung ultrasound in ambulatory heart failure patients. EurHeart J 2016;37(15):1244-51.

16. Platz E, Merz AA, Jhund PS, Vazir A, Campbell R, McMurray JJ. Dynamic changes and prognostic value of pulmonary congestion by lung ultrasound in acute and chronic heart failure: a systematic review. Eur $\mathrm{J}$ Heart Fail 2017;19(9):1154-1163.

17. Dwyer KH, Merz AA, Lewis EF, Claggett BL, Crousillat DR, Lau ES, et al. Pulmonary congestion by lung ultrasound in ambulatory patients with heart failure with reduced or preserved ejection fraction and hypertension. J Card Fail 2018;24:219-226.

18. Gargani L, Pang PS, Frassi F, Miglioranza MH, Dini FL, Landi P, et al. Persistent pulmonary congestion before discharge predicts rehospitalization in heart failure: a lung ultrasound study. Cardiovasc Ultrasound. 2015;13:40

19. Maw AM, Hassanin A, Ho PM, Mclnnes MDF, Moss A, Juarez-Colunga E, et al. Diagnostic accuracy of point-of-care lung ultrasonography and chest radiography in adults with symptoms suggestive of acute decompensated heart failure: a systematic review and meta-analysis. JAMA Netw Open. 2019; 2(3):e190703.

20. Gargani L, Frassi F, Soldati G, Tesorio P, Gheorghiade M, Picano E. Ultrasound lung comets for the differential diagnosis of acute cardiogenic dyspnoea: a comparison with natriuretic peptides. Eur J Heart Fail. 2008; 10:70-7.

21. Russell FM, Ehrman RR, Ferre R, Gargani L, Noble V,Rupp J, et al. Design and rationale of the $B$-lines lung ultrasound guided emergency department management of acute heart failure (BLUSHED-AHF) pilot trial. Heart Lung. 2019:48;186-92.

22. ClinicalTrials.gov [Internet]. Bethesda (MD): National Library of Medicine (US). 2000 Feb 29. Identificador NCT03613779, Lung Ultrasound Guided Therapy in Heart Failure (CLUSTER-HF); 2018 Aug 3 [citado 2018 Nov 14]; 5 páginas]. Disponible en: https://clinicaltrials.gov/ct2/show/NCT03613779. 